\title{
Diseño e implementación de un sistema de control para un horno de crisol
}

\author{
Quiroga Josué $^{1}$, Flores Evelyn ${ }^{2}$, Coba Alfredo ${ }^{3}$ y Jeysson Tapia ${ }^{4}$. \\ josueesteban_12@hotmail.com ${ }^{1}$, eflores.ynmr@gmail.com², alfredocoba@mail.com³, \\ jeyssontap@hotmail.com ${ }^{4}$. \\ https://orcid.org/0000-0001-6819-56743 ${ }^{1}$, https://orcid.org/0000-0002-7425-9360², \\ https://orcid.org/0000-0002-3143-89593, https://orcid.org/0000-0003-4859-76623. \\ Quito-Ecuador
}

Recibido (03/07/20), Aceptado (21/07/20)

\begin{abstract}
Resumen: Este trabajo presenta el diseño de un sistema de control de temperatura, y su proceso de implementación electrónica en un horno de crisol para fundir aluminio. El sistema permite el control de un quemador a gas, cuya llama calienta el horno permitiendo fundir el material. Se incorpora una interfaz gráfica para el ingreso y visualización de los valores de temperatura que se requieren en la cámara del horno. El sistema permitió facilitar el manejo del horno e incorporar la visualización de la temperatura
\end{abstract}

Palabras Clave: Horno a gas, control de temperatura, fundición de aluminio, HMI.

\section{Design and implementation of a control system for a crucible furnace}

\begin{abstract}
This work presents the design of a temperature control system, and its electronic implementation process in a crucible furnace to melt aluminum. The system allows the control of a gas burner, whose flame heats the oven, allowing the material to melt. A graphical interface is incorporated for entering and displaying the temperature values required in the oven chamber. The system made it easier to operate the oven and incorporate the temperature display.
\end{abstract}

Keywords: Gas oven, temperature control, cast aluminum, HMI. 


\section{I.INTRODUCCIÓN}

\section{A.Hornos de Fundición en Aluminio}

La fundición es el proceso en el cual se cambian las propiedades de un metal mediante procesos de calentamiento y enfriamiento, específicamente en nuestro caso se cambiará de estado sólido a liquido el aluminio a una temperatura de 660[1].

Las etapas de fundición son: (1) Calentamiento, (2) Permanencia, (3) Enfriamiento. En la etapa de calentamiento, se eleva la temperatura del aluminio hasta alcanzar su punto de fusión. La permanencia mantiene la temperatura a fin de lograr la fundición completa del aluminio y garantizar su cambio de estado sólido a líquido. En la etapa final de enfriamiento, se disminuye la temperatura del aluminio y regresa a su estado sólido, en la figura 1 se presenta la gráfica de las etapas de los tratamientos térmicos. [2].

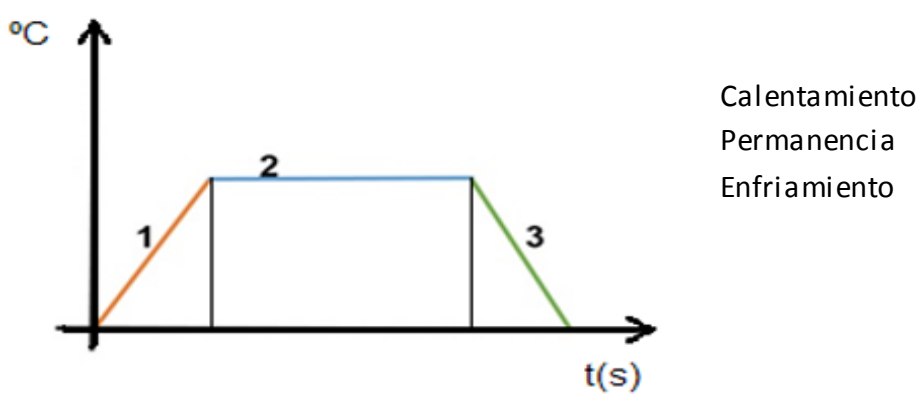

Fig. 1. Gráfica de etapas de fundición.

El aluminio es un metal no ferroso que posee poca resistencia mecánica, se puede formar varias aleaciones para aumentar su resistencia. [3] Un horno de crisol mantiene el calor confinado, además, posee una alta potencia de fusión, evacua los gases a través del crisol y soporta un rango de temperatura de 30 a 3300 grados centígrados. [4]

El quemador a gas es un equipo que se alimenta de un flujo de gas y al activar un electrodo se produce una chispa que genera la llama que elevara la temperatura del horno de crisol y de esta manera fundir el aluminio. [5] Para medir la temperatura, se emplean termocuplas tipo K usadas en aplicaciones industriales como fundición de metales: aluminio, cobre, plata y permiten la medición en un rango de 0 a $1300^{\circ}$ grados centígrados. [6]

\section{B.Sistemas de Control en Hornos de Fundición}

Se han presentado avances en el área del control de hornos de fundición, basada en la tecnología de comunicación y transmisión de señales [7]. Algunos sistemas emplean relés programables, PLCs o incluso sistemas embebidos o informáticos para el control del proceso de fundición. Es indispensable para este proceso el uso de sensores [8], sean estos de temperatura, presión, nivel, caudal y demás variables que pueden considerarse; además. Para el control de dispositivos que regulan el paso de fluidos de combustión, se hace necesario en muchos casos, un actuador que varíe el comportamiento de acuerdo con la información proporcionada por el sensor y partiendo de un valor deseado (Set Point).

Teniendo en cuenta el valor deseado y el valor actual de la variable de control se establece el error, el cual es el valor que ingresará al controlador y este mediante cálculos matemáticos establece la señal de salida que controlará el actuador [9]. El controlador es un sistema microprocesador que realiza operaciones matemáticas para definir el valor que deberá compensar el actuador para llegar al valor deseado de la variable, existen varias formas de compensar, los cuales se llaman estrategias de control, entre ellas se tiene controladores ON/OFF, PID, Cascada, Feedforward, SMC, DSMC etc. [10-12].

El controlador ON/OFF es un controlador todo o nada en cual es muy simple de aplicar y trabaja en un rango, alrededor del punto de operación es decir, se pone en $\mathrm{ON}$ hasta alcanzar el valor del rango que está sobre el punto de operación, para acto seguido apagarse (OFF), sin embargo si la variable alcanza el valor menor del rango el controlador se volverá a encender; para sí mantener el valor siempre entre dentro de este rango; este tipo de controladores es muy usado para sistemas en los cuales no se requiere un valor exacto de la variable de salida, además es usado para procesos lentos con son los de calefacción u hornos, pues son sistemas lentos que conservan el calor, se 
pueden manejar en rangos sin problema, además de que no necesitan manejar exactitud en la variable; adicionalmente otra ventaja de este tipo de control es no necesita un sistema procesado con alta capacidad de procesamiento pues consume una cantidad de recursos informáticos al ser implementado.

Los controladores de tipo PID, son los más distribuidos en la industria cuando se desea que la variable de control oscile de forma muy cercana al Set Point, pero para esto se debe tener un elemento final de control tipo proporcional, lo cual implica un costo adicional, pero con este elemento se llegará con exactitud y de forma eficiente al punto de operación deseado, puesto que si se tiene elementos de tipo todo o nada, este controlador no presentará la eficiencia que se requiere pues la salida de un PID e un valor proporcional al error, el cual irá disminuyendo con forme se aproxime al valor deseado.

Los controladores cascada, se basan en dos lazo de control embebidos, es decir uno dentro del otro, siendo el lazo interno la variable más rápida y el lazo externo la variable más lenta y la que es de interés para el proceso, este tipo de controladores se aplican cuando se quiere controlar una variable de tal forma que si existiese una perturbación fuerte dentro del sistema, la variable principal no se vea afectada en absoluto; sin embargo al ser dos o más controladores, uno dentro de otro, se necesitan igual número de sensores, para captar la variable a controlar de cada lazo, lo cual implica un costo mayor. Los controladores Feedforward, se aplican cuando podemos medir una perturbación dentro del sistema a fin de subsanarla y que la variable de interés no se vea afectada, paro para aplicarlo al igual que en el caso anterior se necesita otro sensor adicional al de la variable de interés. El costo de la aplicación de estos dos tipos de controladores está justificado cuando el proceso en general se ve mayoritariamente afectado por un pequeño cambio en la variable a controlar.

Los controladores SMC, MPC, GPC,LQR y DSMC, forman parte de una serie de controladores denominados robustos o modernos, los cuales si bien tiene una respuesta muy eficiente en varios entornos e incluso no solo funcionan en un rango alrededor el punto de equilibrio como los PID, Cascada y Feedforward; implican una carga computacional muy alta, se basan en modelos complejos, además de que sintonizarlos es una tarea muy extenuante; por lo cual a nivel industrial son muy poco usados e incluso algunos aún solo se siguen manejando de forma académica.

\section{II.DESARROLLO}

El presente desarrollo, toma en cuenta el aspecto de diseño de control, el diseño electrónico y el diagrama de flujo de funcionamiento requerido para el control sistema, como punto de partida se ocupó el equipo ya existente en un laboratorio de Ingeniería Mecánica, que posee un quemador a gas de marca Weishaupt y un horno de crisol

El horno de la figura 2, funcionaba de manera manual y requería de un tiempo considerable de espera para que se enfrié el quemador y apagar el sistema. Esto producía pérdida de tiempo para el operario y un uso ineficiente del equipo ya que no se conocía la temperatura del horno. Por tanto, se presentó la necesidad de incorporar un sistema de control automático para el encendido y apagado. Además, era indispensable visualizar la temperatura en el proceso. La figura 2 presenta el horno a intervenir, junto con sus componentes.

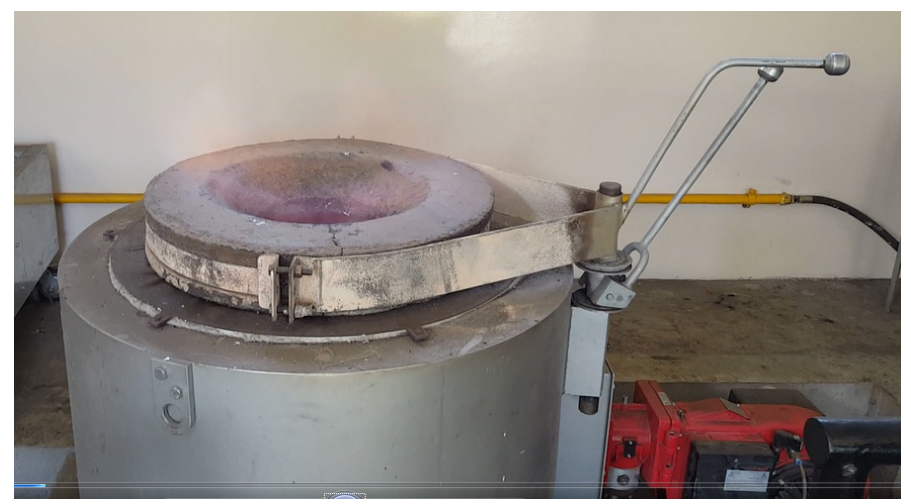

Fig. 2 Esquema de los componentes del horno crisol

A falta de un sensor de temperatura y de una interfaz hombre máquina, se incorporó dos termocuplas tipo K, para la medición de este parámetro en un rango entre $21^{\circ}-750^{\circ} \mathrm{C}$, cuyo valor superior corresponde a un punto 
por sobre la temperatura de fusión del aluminio $\left(660^{\circ}\right)$. Además, se implementó una pantalla LOGO TDE para la monitorización del proceso y se le proporcionó una señal digital para su control, con el empleo de un PLC LOGO de marca SIEMENS.

\section{A.Diseño de Sistema de control}

Se propone realizar un sistema de control ON/OFF porque el proceso de fundición con quemador a gas no necesita rapidez en la etapa de calentamiento. Se necesita mantener la temperatura del horno en la etapa de permanencia, donde se produce el cambio de estado del aluminio de solido a líquido. En la figura 3. se muestra un diagrama de bloques del control lazo cerrado del proceso de fundición.

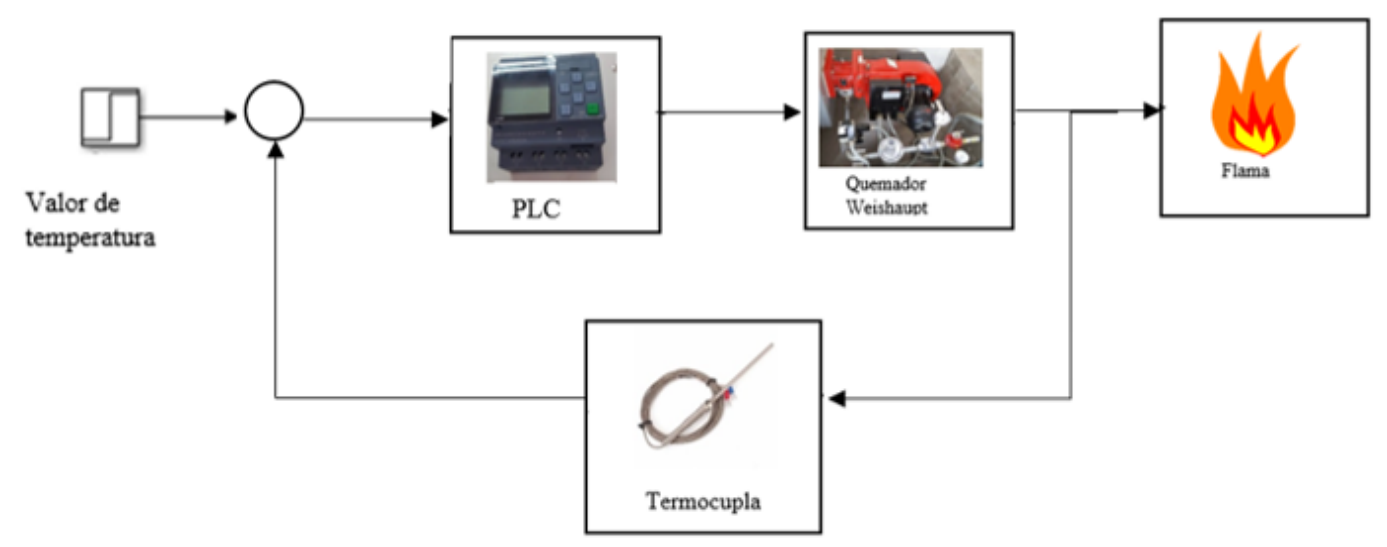

Fig. 3 Diagrama del sistema de control propuesto.

\section{B.Diseño Electrónico}

El diseño electrónico de control se utilizó un PLC LOGO, pantalla Logo LTDE, módulo de adquisición analógico, fuente de alimentación LOGO, termocuplas tipo K y 2 botones de accionamiento.

En el módulo analógico se utilizaron las direcciones: IW0.0, IW1.1, en el PLC LOGO se utilizarán las salidas: Q0.0, Q0.1, Q0.2, Q0.3 y las entradas I0, I0.1. En la figura 4 se presenta el diagrama eléctrico de conexión donde intervienen todos los componentes.

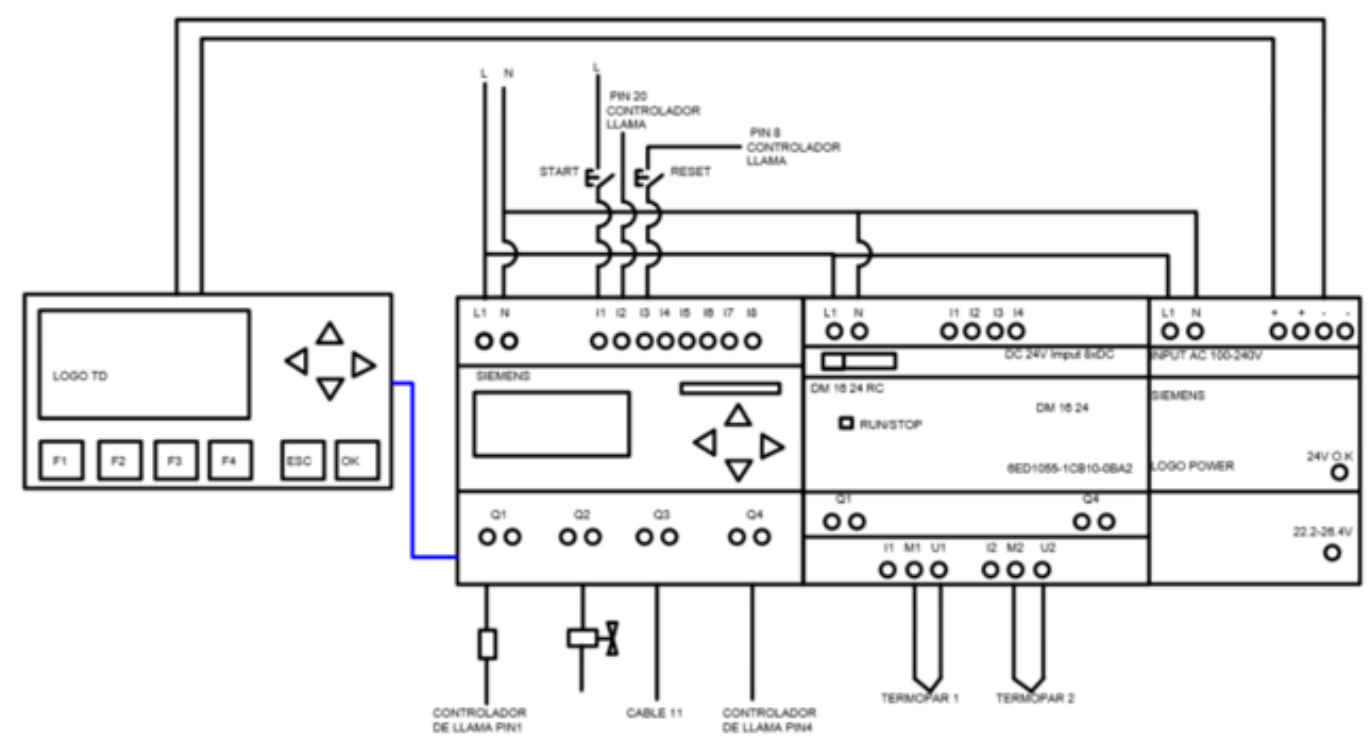

Fig. 4 Diagrama eléctrico de control 
La termocupla tipo K al ser expuesta al calor del horno de crisol, presentará una variación de voltaje en el orden de los microvoltios, que no puede ser registrada por el controlador de manera directa por esta razón, se acondiciona la señal de la termocupla aumentando la ganancia de la salida en $1000 \%$ y obtener un valor de voltaje en el orden de los milivoltios.

En la figura 5. se muestra el diseño del circuito de acondicionador de la señal obtenida por la termocupla.

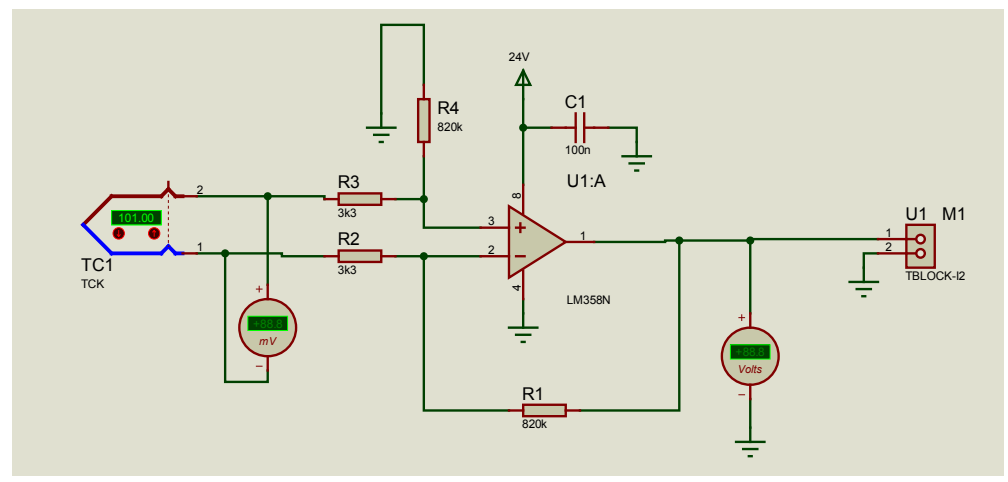

Fig. 5 Simulación del circuito de acondicionamiento.

\section{C.Diagrama de flujo del sistema de control propuesto}

El proceso inicia cuando el usuario ingresa el valor de temperatura llamado consigna, posteriormente esta señal pasa a un controlador lógico programable el cual activa al quemador y produce una llama la cual incrementará la temperatura del horno para fundir el aluminio, las dos termocuplas ubicadas en el horno van a medir la temperatura. Este dato es comparado con el valor de consigna ingresado por el usuario, de esta manera se obtiene el error que

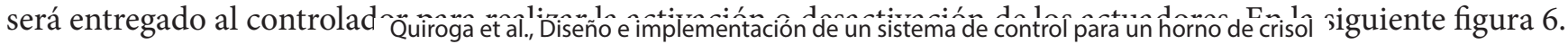
se muestra diseño del algoritmo en diagrama de tlujo a ser implementado.

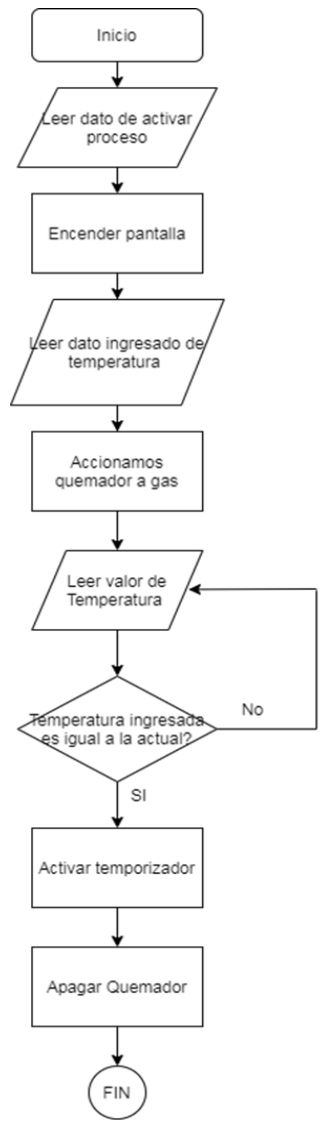

Fig. 6. Algoritmo de funcionamiento 


\section{III.METODOLOGÍA}

Primeramente se realizó una caracterización de laboratorio de mecánica identificando todos los componentes como se muestra en la figura 7, después se analizó el sistema de control a implementar, se seleccionó el equipamiento que cumpla con las necesidades.

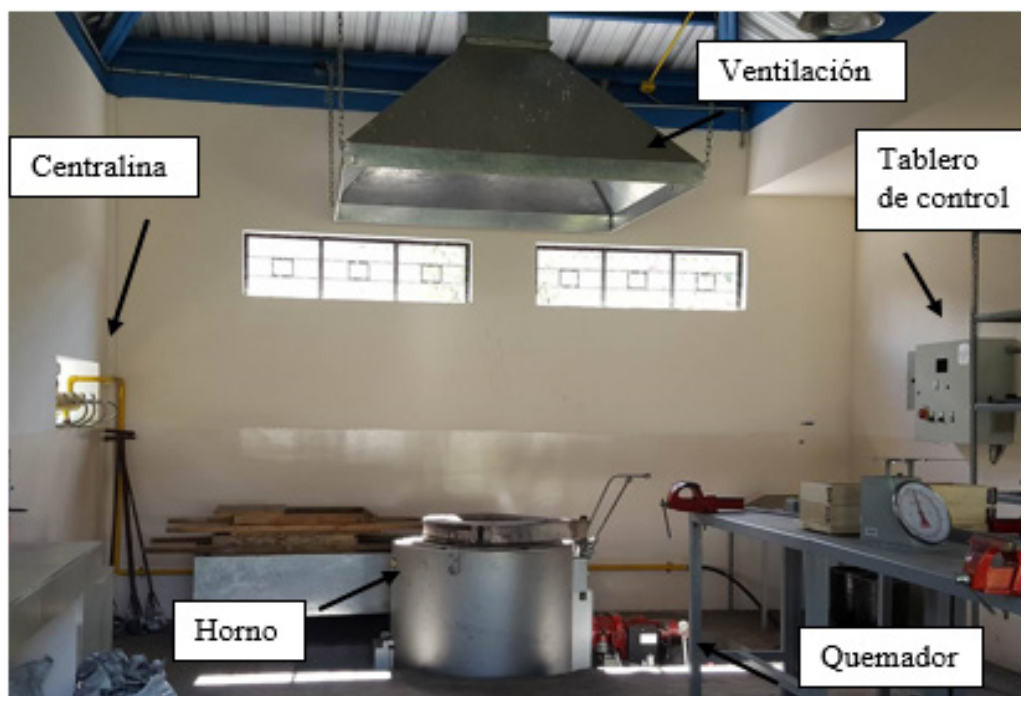

Fig. 7. Laboratorio de fundición, Ingeniería Mecánica.

En la siguiente etapa se diseñó el circuito eléctrico de control utilizando el paquete informático Proteus y se realizó la programación del PLC LOGO en el software LOGO Soft Comfort V8. Para finalmente implementar el circuito de control como se muestra en la figura 8.

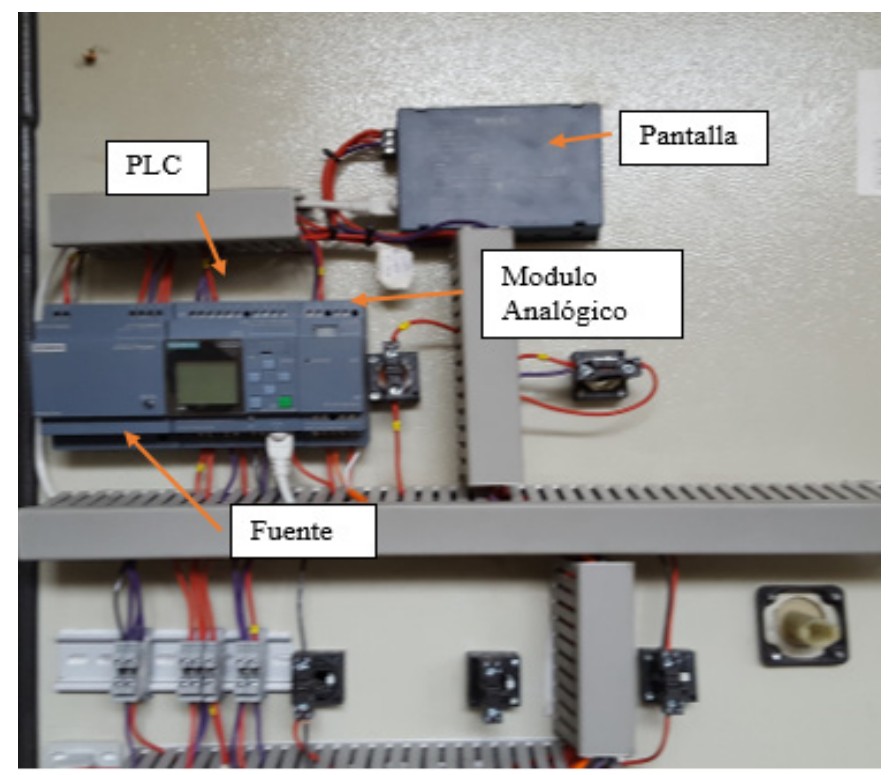

Fig. 8. Laboratorio de fundición, Ingeniería Mecánica.

\section{IV.RESULTADOS}

Una vez realizada la programación del PLC e la implementación del diseño electrónico se visualiza en la figura 9, la pantalla principal del sistema HMI donde el usuario ingresa el valor de temperatura deseada en grados centígrados. La temperatura se setea en un rango de $201^{\circ}$ hasta $1000^{\circ} \mathrm{C}$. Para incrementar la temperatura se debe presionar la tecla ESC y la flecha de la derecha, para disminuir la temperatura se debe presión la tecla ESC y la flecha de abajo y para iniciar el proceso de fundición se debe presionar la tecla F1. 


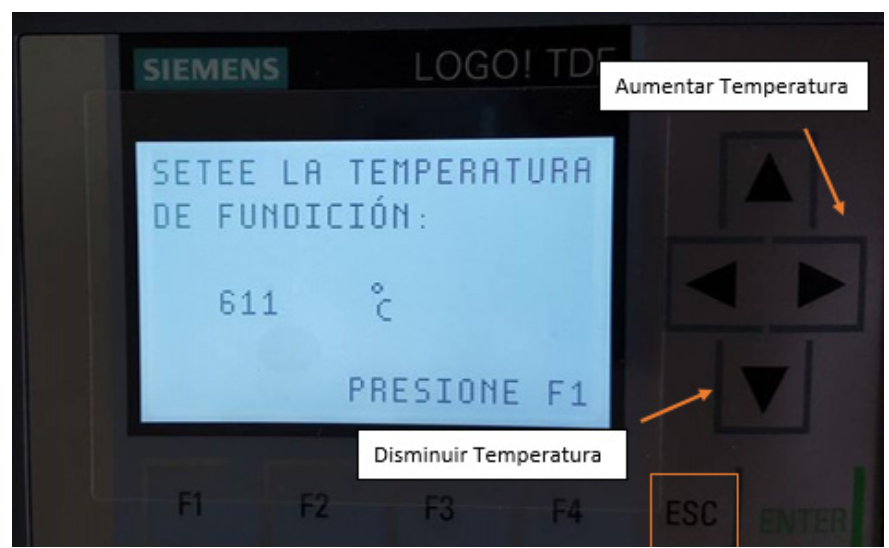

Fig. 9. Monitoreo de temperatura en tiempo real

Después de iniciar el proceso de fundición, la temperatura del horno se incrementará hasta el valor establecido. En la figura 10. se muestra la pantalla de monitorización donde se indican los valores en barras de la temperatura en tiempo real obtenida por la termocupla, si se desea detener el proceso se debe presionar la tecla F2.

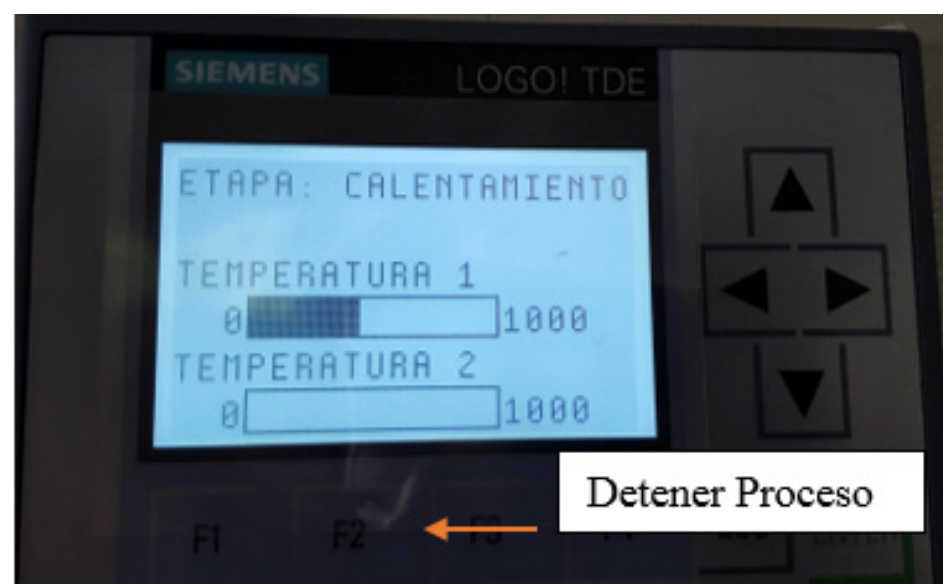

Fig. 10 Monitoreo de temperatura en tiempo real

Si en el proceso de fundición existiera alguna falla en los actuadores o sensores, el controlador detendrá el proceso y se visualizará en la pantalla "error de conexiones" como se muestra en la figura 11, lo que indica al usuario que debe revisar las conexiones.

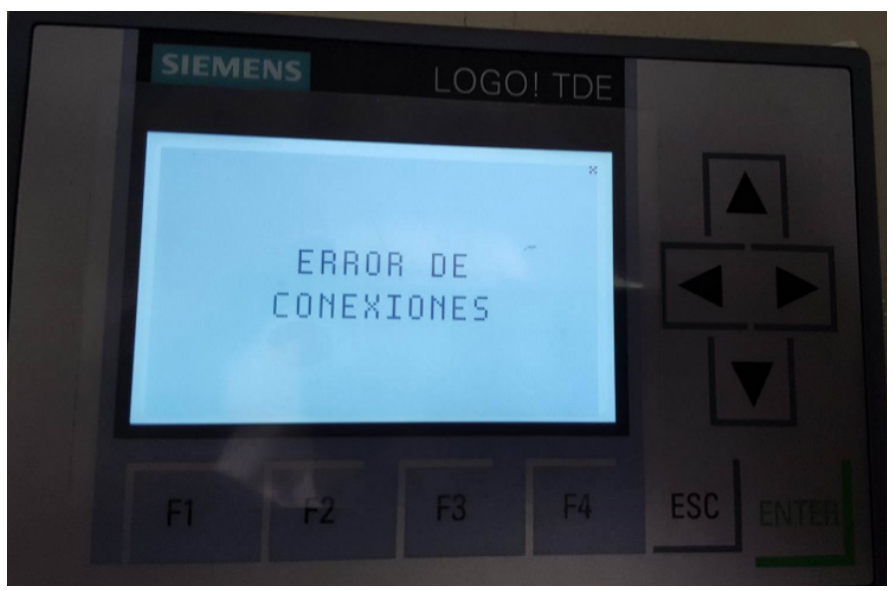

Fig. 11 Alarma por error en conexiones 
Después de finalizar la implementación del sistema de control se fundió el aluminio a una temperatura de $750^{\circ} \mathrm{C}$ en 1 hora $05 \mathrm{~min}$, además, el horno descendido a $200^{\circ} \mathrm{C}$ para su apagado automático del quemador en un tiempo de 3 horas $30 \mathrm{~min}$. Se observó que el color de llama producida por la mezcla es azul, en la figura 12 se muestra la gráfica temperatura del horno vs tiempo de la prueba realizada.

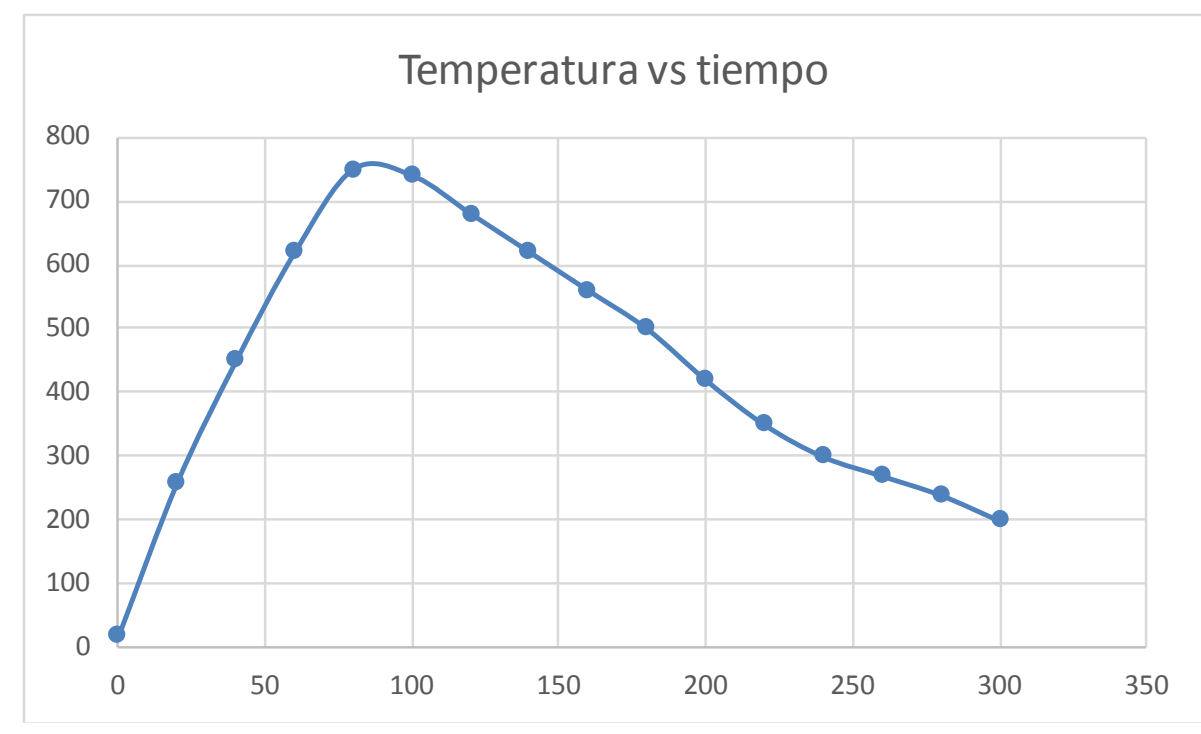

Figura 12.. temperatura vs tiempo

Posterior a las pruebas de funcionamiento se muestra en la figura 13. el horno del laboratorio de Mecánica fundiendo aluminio correctamente.

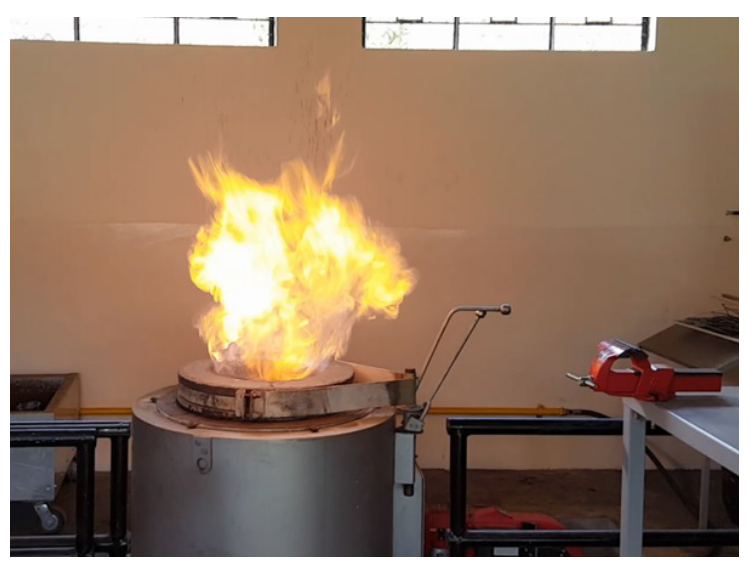

Fig. 13 temperatura vs tiempo

\section{V.CONCLUSIONES}

- La implementación del sistema automático fue exitosa debido a que controló el accionamiento y desactivación del quemador a gas en función a la temperatura seteada por el usuario, lo que conlleva un mejor uso del proceso.

- Gracias al circuito de acondicionamiento implementado para la obtención de los valores de las termocuplas tipo K se pudo realizar la programación para el control del sistema y permitió visualizar en la pantalla LOGO el valor de la temperatura del horno.

-La implementación del sistema de control y la interfaz hombre maquina permitió que el operario del horno monitoree de manera más eficiente y amigable los valores de temperatura.

-En la etapa enfriamiento del horno el operario debía esperar que la temperatura desciende a $200^{\circ} \mathrm{C}$. para apagar el quemador a gas, con la implementación del control automático se independizo al usuario de estar pendiente del proceso. 


\section{VI.APÉNDICE}

Los apéndices, si son necesarios, aparecen antes del reconocimiento.

\section{VII.RECONOCIMIENTO}

Use el título singular aun cuando usted tiene muchos reconocimientos. Evite las expresiones como "Uno de nosotros (S.B.A.) gustaría agradecer... " En cambio, escriba "F. A. agradecimientos del autor... ." reconocimientos a patrocinador y de apoyo financieros se ponen en la nota a pie de página de la primera página sin numerar.

\section{REFERENCIAS}

[1]M. Jami, E. Suntaxi, C. Torres., "Diseño y construcción de un horno crisol para fundición de aluminio con una capacidad de $15 \mathrm{~kg} / \mathrm{h}$ a una temperatura de $800^{\circ} \mathrm{C}$ utilizando GLP," Quito: Universidad Politécnica Salesiana, 2014. [2]V. Rodas., "Diseño e Implementación de un Sistema de Control Automático para seis hornos. Quito: Escuela Politécnica Nacional”, 2015.

[3]G. Earnshaw., (2008), Química de los elementos (Chemistry of the Elements). [Online], Available: http://www. lenntech.es/periodica/elementos/al.htm.

[4]G. Nabertherm., (2016). Fundición. Obtenido de Conceptos alternativos de hornos de fundición. [Online], Available: http://www.nabertherm.es/produkte/details/es/giesserei_schmelz-und-warmhalteoefen.

[5]Catálogo, S/N, Weishaupt., Producto: Instalacion sobre quemadores compactos, España.

[6]D. Bedoya, "Diseño de un contrastador de temperatura para termómetros de termocuplas tipo K," Pontifice Universidad Católica del Perú, 2014.

[7]P. González y R. Noriega, "Diseño e Implementación de un Módulo Didáctico para Control de Nivel, Temperatura y Caudal mediante la red de Comunicación de Campo DeviceNet", Sangolqui, Ecuador, may 2012.

[8]A. Creus, Instrumentación industrial, 8a ed. México D.F.: Alfaomega Grupo Editor, S.A. de C.V., 2011.

[9]C. Smith, A. Corripio, Principles and Practice of Automatic Control, 3a ed. Estados Unidos de América, New York: Wiley, 2005.

[10]D. Seborg, D. Mellichamp, T. Edgar, F. Doyle, Process Dynamics and Control, 4a ed. Estados Unidos de América: Wiley, 2016.

[11]J. Tapia, A. Rodas, O. Camacho., "Comparación de dos enfoques para la enseñanza en Control de Procesos: Simulación versus Implementación,” Revista Risti, vol. 23, pp. 206-219, 2019.

[12]N.D. Tapia,. (2014). Transferencia de calor, [Online], Available http://www.monografias.com/trabajos15/ transf-calor/transf-calor.shtml

\section{RESUMEN CURRICULAR}

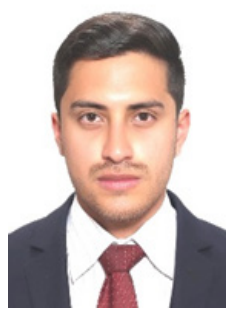

Josué Quiroga, Ingeniero electrónico de la Escuela Politécnica del Ejército, apasionado por la investigación y desarrollo de equipos especiales, con amplia experiencia en las áreas de telecomunicaciones e inmótica, asesor de la empresa Electronic Solutions \& Services.

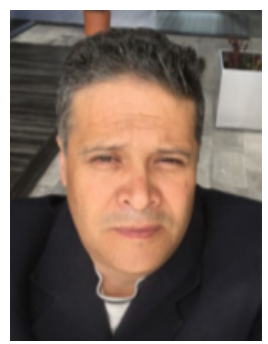

Alfredo Coba, Ingeniero electrónico de la Escuela Politécnica del Ejército, apasionado por la investigación y desarrollo de equipos especiales, con amplia experiencia en las áreas de telecomunicaciones e inmótica, asesor de la empresa Electronic Solutions \& Services. 


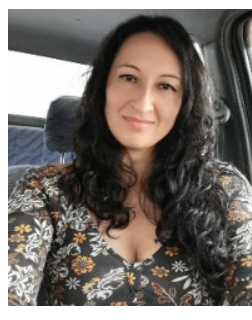

Evelyn Flores, Estudiante de Ingeniería en Redes y Telecomunicaciones de la Universidad de las Américas, especialista en optimización de sistemas neumáticos y diseño e instalación de redes de aire comprimido a nivel industrial. Quito Ecuador

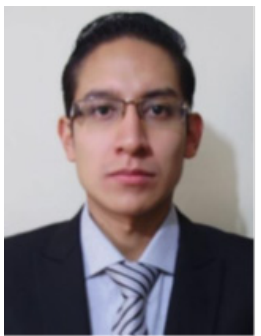

Jeysson Tapia, Ingeniero en Electrónica y Control de la Escuela Politécnica Nacional, apasionado por la tecnología, la automatización y más aún por la instrumentación biomédica, con experiencia en instrumentación, control y manejo de sensores. Quito - Ecuador. 